\title{
CHANGES IN THE AMYGDALA AMINO ACID MICRODIALYSATE AFTER CONDITIONING WITH A CUE ASSOCIATED WITH ETHANOL
}

\author{
Etienne Quertemont, Jessica de Neuville and Philippe De Witte
} Université Catholique de Louvain, Biologie du Comportement, Belgium

The excitatory amino acid neurotransmission within the amygdala has been implicated in learning associations between external stimuli and intrinsic reward values such that it may play a key role in conditioned drug effects. In the present studies, the responses of the excitatory amino acids, aspartate and glutamate together with the neuromodulatory sulphonated amino acid taurine, within the basolateral amygdala, to an odor cue repeatedly associated with acute ethanol injections $(2 \mathrm{~g} / \mathrm{kg}, \mathrm{IP})$ have been investigated by a microdialysis technique combined with HPLC-EC analysis. After presentation of the ethanol-conditioned stimulus, a single IP saline injection induced an immediate and significant increase in the taurine microdialysate content which could be related to the neuromodulatory action of taurine. Furthermore, when the conditioned stimulus was combined with the ethanol injection $(2 \mathrm{~g} / \mathrm{kg}$, IP), significant increases in both taurine and glutamate microdialysate content were observed and indicated a learned compensatory response to counteract the acute effects of ethanol. These results demonstrate that changes in amygdala extracellular glutamate and taurine concentrations can be conditioned to ethanol-associated stimuli and are therefore probably implicated in the phenomenon of environmental-dependent tolerance to ethanol. 